\title{
Heuristic Spectrum Assignment Algorithm in Distributed Cognitive Networks
}

\author{
Li Yu, Cong Liu, Zuhao Liu, Wenyu Hu \\ Huazhong University of Science and Technology, Department of Electronic Information Engineering, \\ Wuhan National Laboratory for Optoelectronics, Div Commun and Intelligent Networks, Wuhan, China \\ E-mail:hustlyu@mail.hust.edu.cn, \{liuconggg,liuzuhao,babywinnerhu\}@gmail.com \\ Received December 14, 2009; revised February 5, 2010; accepted February 10, 2010
}

\begin{abstract}
Cognitive radio is an exciting emerging technology that has the potential of dealing with the urgent requirement and scarcity of the radio spectrum. Although having multiple radio interfaces and available spectrum bands can generally increase the effective throughput, a problem arises as to what the best strategy to dynamically assign available bands to secondary users for maximizing throughput by minimizing the interference, and what the best scheme to allocate the spectrum holes to unlicensed users to maximize the fairness. This paper presents a distributed and heuristic spectrum assignment algorithm for multi-radio wireless cognitive networks in a cognitive network environment. The proposed algorithm (Fairness Bargaining with Maximum throughput, FBMT) considers the problems including system throughput and the fairness. Extensive simulation studies in 802.11 based multi-radio cognitive networks have been performed. The results indicate that the proposed algorithm can facilitate a large increase in network throughput and acquire a good fairness performance in comparison with a common spectrum assignment mechanism that is used as a benchmark in the literature.
\end{abstract}

Keywords: Cognitive Network, Distributed Spectrum Assignment, Throughput, Fairness, FBMT

\section{Introduction}

As wireless technologies continue to grow, more and more spectrum resources will be needed. Within the current spectrum regulatory framework, however, all of the frequency bands are exclusively allocated to specific services, and no violation from unlicensed users is allowed. A recent survey of spectrum utilization made by the Federal Communications Commission (FCC) has indicated that the actual licensed spectrum is largely underutilized in vast temporal and geographic dimensions [1]. For instance, a field spectrum measurement taken in New York City has shown that the maximum total spectrum occupancy is only $13.1 \%$ from $30 \mathrm{MHz}$ to $3 \mathrm{GHz}$ $[2,3]$. Similar results, obtained in the most crowded area of downtown Washington, D.C., indicated occupancy of less than $35 \%$ of the radio spectrum below $3 \mathrm{GHz}$. Moreover, the spectrum usage varies significantly in various time, frequency, and geographic locations.

\subsection{Spectrum Opportunity}

Spectrum segments in a licensed band that are currently unused by its primary users (PU) are referred to as the spectrum opportunity/holes. Spectrum holes appear as useable spectrum bands to a secondary user (SU) with respect to the licensed band in question. For a secondary user, given its locations, a set of spectrum holes can be available at a given time. Such a set of available bands are referred to as the spectrum opportunity of the secondary user.

Spectrum utilization can be improved significantly by allowing a secondary user to utilize this spectrum opportunity when the primary user is absent. Cognitive radio (CR), as an agile radio technology, has been proposed to promote the efficient use of the spectrum [4]. By sensing and adapting to the environment, a $\mathrm{CR}$ is able to fill in spectrum holes and serve its users without causing harmful interference to the licensed user.

Amid these usage trends, it is desirable to introduce dynamic spectrum allocation strategies so that the unused segments of a licensed band which owned by its primary users can be used by unlicensed or secondary users. A fixed spectrum policy was sufficient in the past but with the increasing disparity in utilization rate between the licensed and unlicensed bands, dynamic spectrum alloca- 
tion policies are needed.

\subsection{Related Work}

Dynamic spectrum allocation strategies enable secondary user to sense local usable bands (including the bands without license), and to use them by some certain rules/ algorithms without interference to primary users. According to the difference of structures of networks, the algorithms can be divided into two categories: the centralized and the distributed. In centralized spectrum allocation algorithms, due to higher computing complexity, the centralized control devices become a bottle-neck because they are not competent for such complex computing work. So the distributed algorithms are much better than the centralized algorithm in spectrum assignment. Most distributed algorithms adopt heuristic assignment methods, in which algorithm's astringency is a very important target, and the higher the astringency of algorithms is, the better the algorithm can adapt to the timevarying systems. Beside astringency, in [5-7], Zheng et al. put forward other two basic targets, Max-SumBandwidth (MSB) and Max-Min-Bandwidth (MMB), these targets are used to describe the improvement of throughput that the algorithm brings to system. In [8], Cao et al. proposed a FBFP (fairness bargaining with feed poverty) algorithm to Ad Hoc networks, in this algorithm, secondary users are divided into different groups based on their regions. Simulation result present that this algorithm can decrease the computing complexity effectively but increase the system cost because it needs extra packet to set up and maintain the groups. In $[9,10]$, Wang and Liu proposed several algorithms which based on the max-spectrum-employ rate and max- fairness, but the throughput of system is absent in their consideration. In [11], by using the time-continuous Markov

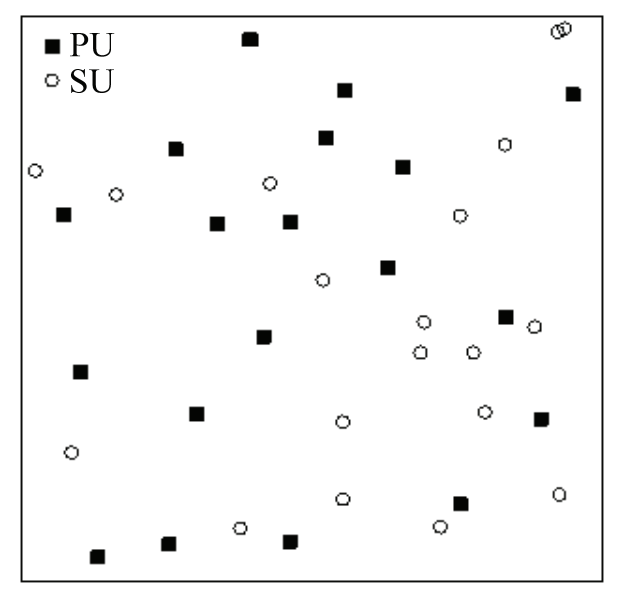

(a) chain to model the spectrum access, Xing et al. proposed a random spectrum access algorithm, but this algorithm is only applicable in simple systems which consist of fewer primary users and secondary users.

Motivated by this, considering both the fairness and throughput, we bring forward a heuristic algorithm called Fairness Bargaining with Maximum Throughput (FBMT). The simulation will demonstrate that FBMT not only contribute to promote the systems fairness, but also improve the system throughput.

The rest of this paper is organized as follows. In Section 2, a completely distributed system model will be briefly reviewed, and a mathematic model will be designed for the spectrum assignment and coordination process. In Section 3, we give the detailed description of FBMT. We evaluate the performance of FBMT by our simulation in Section 4. Finally, we conclude the paper.

\section{Preliminary}

\subsection{Wireless Cognitive Network Architecture}

First, we characterize a distributed system model: $M$ primary users and $N$ secondary users are randomly distributed in an $X \times Y$ area (Figure 1(a)), secondary can using a certain signal detection techniques such as matched filter detection, energy detection or cyclostationary detection to get the usable spectrum opportunity. In the system, available spectrums are divided into $K$ completely orthogonal bands. We assume that these bands are symmetric, which means that these bands have the same bandwidth, meanwhile, have the same spectrum quality and the spectrum availability. We also assume that the interference between two nodes only rest with the relative distance of theirs, all of the primary users and

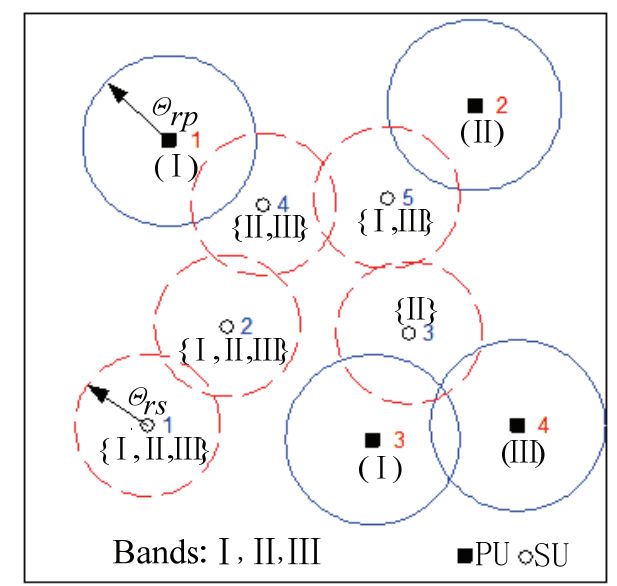

(b)

Figure 1. (a) Sketch map of the cognitive radio system; (b) An example of cognitive radio system. 
secondary users utilize the omni-antenna, we define the transmission radius of primary users and secondary users are $\Theta_{r p}(i, k)$ and $\Theta_{r s}(j, k)$ respectively, where $i, j$ and $k$ denote the serial number of the primary user, secondary user and spectrum bands respectively. If the distance between the primary user and secondary user is greater than $\Theta_{r p}(i, k)+\Theta_{r s}(j, k)$, the secondary user will not conflict with primary user when they select the same band to propagate data simultaneously. In order to analyze simply, we assume that all of the primary users have the same transmission range (express as $\left.\Theta_{r p}\right)$, and all of the secondary users have the same transmission range (express as $\Theta_{r s}$ ). Moreover, we define that the node $i$ and $j$ are neighbours if their transmission coverage area is overlapped with each other.

Figure 1(b) depicts generic wireless cognitive network architecture. The network consists of four primary users and five secondary users; there are three orthogonal wireless bands which are denoted by I, II and III. From the figure we can see that the transmission range of $\mathrm{SU}_{3}$ conflicts with the transmission of $\mathrm{PU}_{3}$ and $\mathrm{PU}_{4}$, which occupy the band I and III respectively. Definitely, $\mathrm{SU}_{3}$ can only utilize the spectrum II to transmit data. As for $\mathrm{SU}_{1}$, it can use the spectrum I, II and III without interference to any PUs; As for $\mathrm{SU}_{2}$, it also can use the spectrum I, II and III, meanwhile, $\mathrm{SU}_{2}$ and $\mathrm{SU}_{1}$ is neighbour node mutually, in order to avoid the conflict between them, they cannot use the same spectrum to propagate data simultaneously. We need to establish some rules to assign the available spectrums to each secondary user. In this paper, we aim to maximize the system throughput by maximize the total spectrum utilization rate.

\subsection{Definitions}

1) In a network waiting for spectrum assignment, there are $N$ secondary users indexed from 1 to $N$ competing for $K$ spectrum bands indexed 1 to $K$.

2) $S=\left\{s_{i, k} \mid i=1, \ldots, N ; k=1, \ldots, K\right\} \quad$ characterize the per user available spectrum, i.e., spectrum band $k$ is available for user $i$ if $s_{i, k}=1$. Due to differences in user locations, technology employed in different spectrums and user requirements, different users will perceive different available spectrum. In Figure 1(b), we can obtain

$$
S=\left[\begin{array}{lllll}
1 & 1 & 0 & 0 & 1 \\
1 & 1 & 1 & 1 & 1 \\
1 & 1 & 0 & 1 & 1
\end{array}\right]^{T}
$$

3) We also consider that the throughput achieved by different bands is different, depending on the user's environment and the attribute of spectrums. Let

$$
B=\left\{b_{i, k} \mid i=1, \ldots, N ; k=1, \ldots, K\right\}
$$

describe the reward that a user $i$ gets by successfully acquiring available spectrum band $k$, i.e., $b_{i, k}$ represents the maximum throughput that can be acquired (assuming no interference from other neighbours). Let

$$
S_{B}=\left\{s_{i, k} \cdot b_{i, k}\right\}_{N \times K}
$$

denote the bandwidth weighted available spectrum.

4) We characterize interference between two competing users by a constraint set. Let

$$
C=\left\{c_{i, j, k} \mid c_{i, j, k} \in\{0,1\}\right\}_{N \times M \times K}
$$

represent the constraint, where if $c_{i, j, k}=1$, users $i$ and $j$ can cause interference if they use the spectrum band $k$ simultaneously.

5) We define a valid spectrum assignment

$$
A=\left\{a_{i, k} \mid a_{i, k} \in\{0,1\}\right\}_{N \times K}
$$

where $a_{i, k}=1$ denotes that spectrum band $k$ is assigned to user $i, A$ satisfies all the constraints defined by $C$, that is:

$$
a_{i, k} \cdot a_{j, k}=0, \text { if } c_{i, j, k}=1 ; i, j \leq N ; k \leq K
$$

Finally, we use $\Lambda_{N, K}$ to denote the set of valid spectrum assignments for a given set of $N$ users and $K$ spectrum bands.

6) We must pay attention that different secondary users have different neighbors in different bands, which means that different secondary users have various influence to system throughput if they are assigned to identical band. We must take this heterogeneity into consideration in spectrum allocation process, we define the gain matrix

$$
R=\left\{r_{i, k} \mid i=1, \ldots, N ; k=1, \ldots, K\right\}
$$

to describe this impact, where $r_{i, k}=b_{i, k} / \varphi_{i, k}, \varphi_{i, k}$ is the neighbor numbers of user $i$ in spectrum band $k$.

\subsection{Optimization Problems}

The objective of a general resource allocation problem can be defined in terms of a utility function. In spectrum related resource allocation problems, we usually need to solve the optimization problem expressed as follows:

1) Max-Sum-Bandwidth (MSB): This aims to maxi- 
mize the total spectrum utilization in the system. The optimization problem is expressed as:

$$
\max _{A \in \Lambda_{N, K}} \sum_{i=1}^{N} \sum_{k=1}^{K} s_{i, k} \cdot b_{i, k}
$$

2) Max-Min-Bandwidth (MMB): This aims to maximize the bottleneck user's spectrum utilization. The optimization problem can be expressed by:

$$
\max _{A \in \Lambda_{N, K}} \min _{i<N} \sum_{k=1}^{K} s_{i, k} \cdot b_{i, k}
$$

\subsection{Color-Sensitive Graph Coloring Problem}

By mapping each spectrum into a color, the spectrum allocation problem can be simplified as a GMC (Graph Multi-Coloring) problem. First, we define a bidirectional graph $G=\left\{V, E_{C}, S_{B}\right\}$, where $V$ is a set of nodes denoting the users that share the spectrums, $S_{B}$ represents the bandwidth weighted available spectrum as defined in Section 2.2, or the color list at each node, and $E_{C}$ is a set of undirected edges between nodes representing interference constraints between two nodes defined by $C$. For any two distinct nodes $i, j \in V$, an $k$-color edge between $i$ and $j$, is in $E_{C}$ if and only if $c_{i, j, k}=1$. Hence, any two distinct nodes can have multiple colored edges between them. We define the color $k$ specific degree of a node $i$, i.e., $\varphi_{i, k}$ to represent the number of neighbours that are color $k$ mutually constrained with $i$ (those who can not use $k$ if $i$ uses color $k$ ). It is also a relatively good measure of the impact (to neighbours) when assigning a color to a node. The equivalent graph coloring problem is to color each node using a number of colors from its color list, such that if a color $k$ edge exists between any two distinct nodes, they can't be colored with $k$ simultaneously. We name this the Color Sensitive Graph Coloring (CSGC) problem.

\section{Proposed Spectrum Assignment Algorithm}

The optimal coloring problem is known to be NP-hard. In this section, we discuss a set of heuristic based approaches that produce good coloring solutions. In particularly, we extend some of the well-known graph coloring solutions toward our problem settings and optimization goals.

\subsection{The Analysis of Existing Algorithms}

\subsubsection{Collaborative-Max-Sum-Bandwidth Rule}

This rule aims to maximize the sum of bandwidth weighted color usage, corresponding to MSB optimization defined in (2). When a node $i$ is assigned with a color $k$, his contribution to the sum bandwidth in a local neighborhood can be computed as $b_{i, k} / \varphi_{i, k}$ since his neighbors can not use the color. Here $\varphi_{i, k}$ represents the number of $k$ color constrained neighbour of a node $i$ in the current graph. We propose to label the node according to

$$
\begin{gathered}
\text { label }_{i}=\max _{k \in s_{i}} b_{i, k} / \varphi_{i, k} \\
\text { color }_{i}=\arg \max _{k \in s_{i}} b_{i, k} / \varphi_{i, k}
\end{gathered}
$$

where $S_{i}$ represents the color list available at node $i$ at this stage. This rule considers the tradeoff between spectrum utilization (in terms of selecting the color with the largest bandwidth) and interference to neighbours. This rule enables collaboration by taking into account the impact to neighbours. If two nodes have the same label, then the node with lower assigned bandwidth weighted colors will get a higher label.

\subsubsection{Random (RAND) Rule}

Each node generates a random label which is between $\left[0\right.$, window $\left._{i}\right]$, where window $_{i}$ denote the threshold of the node $i$. At the beginning of the allocation, all of the nodes have the same threshold. In an allocation process, if node $i$ get a color, window $w_{i}=$ window $_{i} / 2$, else window $_{i}=$ windo $_{i} \times 2$. In each stage, each node labels itself according to the above labeling rules, and broadcasts the label to his neighbors. A node with the maximum label within his neighborhood gets to grab the color associated with his label and broadcasts the color assignment to his neighbors. After collecting assignment information from surrounding neighbors, each node updates his color list and recalculates the label. This process is repeated until the color list at each node is exhausted or all the nodes are satisfied.

Although RAND can optimize fairness performance in spectrum allocation process, however, it cannot guarantee the network throughput. This will be demonstrated in next section.

\subsection{Fairness Bargaining with Maximum Throughput (FBMT)}

In this section, we describe the Fairness Bargaining with Maximum Throughput algorithm-a distributed heuristic spectrum assignment strategy.

The algorithm is based on the following assumptions:

1) Distributed wireless network architectures.

2) All the primary users have the same transmission range, the same to secondary users.

3) All of the secondary users can detect and utilize all 
of the spectrum holes.

4) Primary users have the preferential right of spectrum bands.

5) Users can communicate with each other by using some mechanism.

In FBMT algorithm, we aim to maximize the sum of bandwidth, corresponding to MSB optimization defined in (2). When a node $i$ is assigned with a color $k$, its contribution to the sum bandwidth in a local neighbourhood can be computed as $r_{i, k}=b_{i, k} / \varphi_{i, k}$. We also search for the fairness in spectrum distribution, defined a fairness factor, which can be expressed by

$$
f_{i, k}(t)=\frac{\sum_{j=1}^{N} 1\left\{d_{i, j} \leq 2 \cdot \Theta_{r s}\right\}}{\sum_{k=1}^{K} s_{i, k}(t-1)} .
$$

where $t$ denotes current allocation times, numerator denotes the neighbour numbers of node $i$ in spectrum band $k$, denominator means the number of bands which is assigned to node $i$ before the $t$-th assignment, $d_{i, j}$ denotes the distance between the node $i$ and node $j$. We define

$$
f_{i, k}(t)=\sum_{j=1}^{N} 1\left\{d_{i, j} \leq 2 \cdot \Theta_{r s}\right\}
$$

if $\sum_{k=1}^{K} s_{i, k}(t-1)=0$.

In FBMT, We propose to label the node according to

$$
\begin{aligned}
& \text { label }_{i}=\max _{k \in s_{i}} r_{i, k} \cdot f_{i, k}(t) \\
& \text { color }_{i}=\arg \max _{k \in s_{i}} r_{i, k} \cdot f_{i, k}(t)
\end{aligned}
$$

where $i=1, \ldots, N, k=1, \ldots, K$. This rule not only considers the tradeoff between spectrum utilization and interference to neighbours, but also considers the fairness in spectrum allocation by introduction of the fairness factor. The Pseudo code of HBTM algorithm is shown in Figure 2 .

\section{Performance}

\subsection{Performance Index}

We often evaluate an algorithm by the throughput and fairness it can achieve in spectrum allocation. The throughput, as defined herein, is the sum of bands each user is assigned, which can be expressed by

$$
\Gamma_{\text {Throughput }}(A)=\sum_{i=1}^{N} \sum_{k=1}^{K} a_{i, k} \cdot b_{i, k}, A \in \Lambda_{N, K}
$$

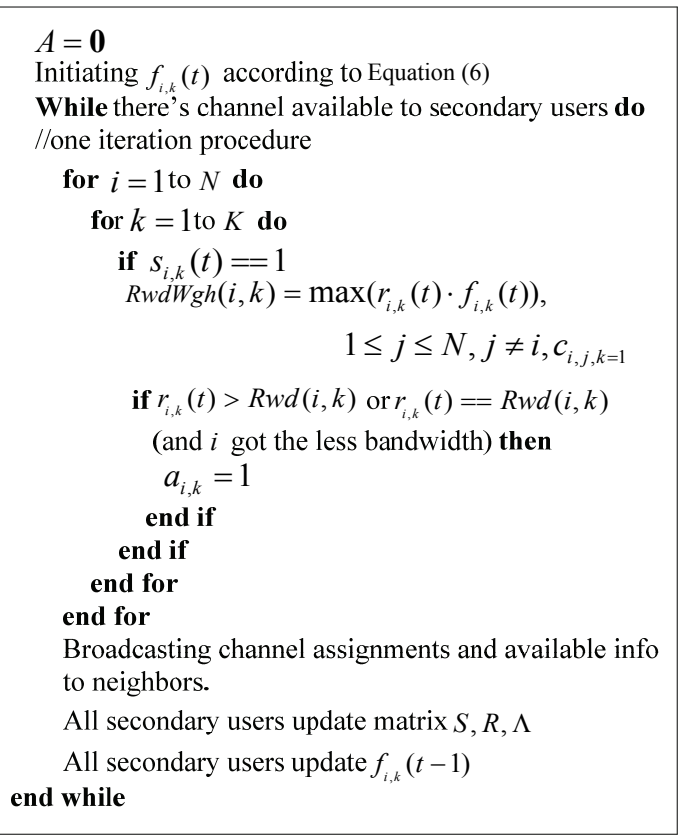

Figure 2. Pseudo code of HBTM algorithm.

In order to analysis the fairness of each spectrum algorithms, we also define the fairness

$$
\Gamma_{\text {fairness }}(A)=\frac{\left(\sum_{i=1}^{N} \sum_{k=1}^{K} a_{i, k} \cdot b_{i, k}\right)^{2}}{\left(N \cdot \sum_{i=1}^{N}\left(\sum_{k=1}^{K} a_{i, k} \cdot b_{i, k}\right)^{2}\right)}, \quad A \in \Lambda_{N \times K}
$$

In (11), we can conclude that $\Gamma_{\text {fairness }} \in[0,1]$, the more closely the $\Gamma_{\text {fairness }}$ approach to 1 , the more impartial the process of spectrum allocation has. We also define

$$
\begin{gathered}
\Gamma_{\text {fairness }}(A)=\left(\sum_{i=1}^{N} \sum_{k=1}^{K} a_{i, k} \cdot b_{i, k}\right)^{2}, A \in \Lambda_{N \times K} \\
\text { if } \sum_{i=1}^{N}\left(\sum_{k=1}^{K} a_{i, k} \cdot b_{i, k}\right)^{2}=0 .
\end{gathered}
$$

\subsection{Simulations}

\subsubsection{Simulation Parameters}

In this section we evaluate the performance of FBMT, We design and implement a prototype system which consists of some primary users and some secondary users, these users are deployed at random in our simulation. Our experiments apply following evaluation metrics:

- The throughput $\Gamma_{\text {Throughput }}$;

- The fairness $\Gamma_{\text {fairness }}$; 
We compared FBMT with a recent CMSB algorithm and RAND algorithm, CMSB represents the Max-bandwidth-based spectrum allocation algorithm, and RAND algorithm is the Max-fairness-based.

In our simulations, we have considered values of $N$ and $M$ ranging from 10 to 35 . The values of $K$ ranging from 10 to 30 , for each situation, we have assigned $b_{i, k}$ with different value (constant or variable). The parameters of simulation circumstance are shown in Table 1 in detail.

\subsubsection{Numerical Result}

Figures 3, 5 and 7 depict the throughput of three algorithms on condition of the various numbers of primary users, secondary users and spectrums, the same with Figures 4, 6 and 8 with the fairness. All the comparisons have different $b_{i, k}$. In (a), $b_{i, k}$ is a constant, while in (b) the value of $b_{i, k}$ is a random distributed in $[0,1]$. In Figures 3, 5 and 7, we can find out if $b_{i, k}$ is identical, the throughput of FBMT is almost equal to CMSB, but larger than RAND; if $b_{i, k}$ is a random number, the throughput of FBMT has $10 \%$ gain compared with CMSB, and has a more gain compared with RAND. In Figures 4, 6 and 8, we can find the FBMT is all square to RAND but better than CMSB in fairness, but the former has the lower throughput relatively.

The simulation result shows that the CMSB, although has a high throughput, but with a low fairness performance; the RAND, has an agreeable fairness performance, but not well in enhancing the throughput. The FBMT, not only succeeds in throughput achieving, but also makes a good fairness performance in spectrum assignment. Relatively FBMT is the best spectrum allocation algorithm in these algorithms.

Figure 3 shows the system throughput of different primary users with CMSB, RAND and FBMT algorithms respectively on the premise of 30 SUs competing 15 channels. We can find that if $b_{i, k}$ is identical such
Table 1. The simulation circumstance.

\begin{tabular}{cc}
\hline Parameter & Value \\
\hline Area & $1.0 \times 1.0$ \\
Transmission range of Pus & 0.25 \\
Transmission range of Sus & 0.15 \\
The number of Pus & $10,15,20,25,30,35$ \\
The number of Sus & $10,15,20,25,30,35$ \\
The number of spectrums & $10,12,14,16,18,20,25$, \\
The simulation time & 30 \\
\hline
\end{tabular}

like $b_{i, k}=1$, the three algorithms almost have the same throughput, while if random, the throughput of FBMT is maximum, CMSB less if primary users are less than 30 , and RAND least. Figure 4 shows the fairness of PUs with three algorithms. We can find that CMSB has much lower performance compared with FBMT and RAND. Moreover, if $b_{i, k}=1$, FBMT has the best fairness performance.

Figures 5 and $\mathbf{6}$ depict the throughput and fairness of secondary users. When there are 20 primary users and 15 channels for secondary users, when $b_{i, k}$ is a random number, the FBMT present the most preferable performance, CMSB less and RAND least. However when $b_{i, k}$ is set 1 , CMSB has better throughput gain. But whether $b_{i, k}$ is confirmed or not, CMSB has the lowest fairness performance.

Figures $\mathbf{7}$ and $\mathbf{8}$ show the results in which the channel number is fixed. If $b_{i, k}$ is a constant, we can find that RAND has the lowest throughput and FBMT the highest. However if $b_{i, k}$ is not fixed, the throughput of three algorithms is almost the same. Also we can find that CMSB still has the lowest fairness performance.

The simulation result reflects that although CMSB has a high throughput, the fairness performance is not satisfying. RAND has an agreeable fairness characteristic but not much well in throughput. However, FBMT can achieve both performances of throughput and fairness.

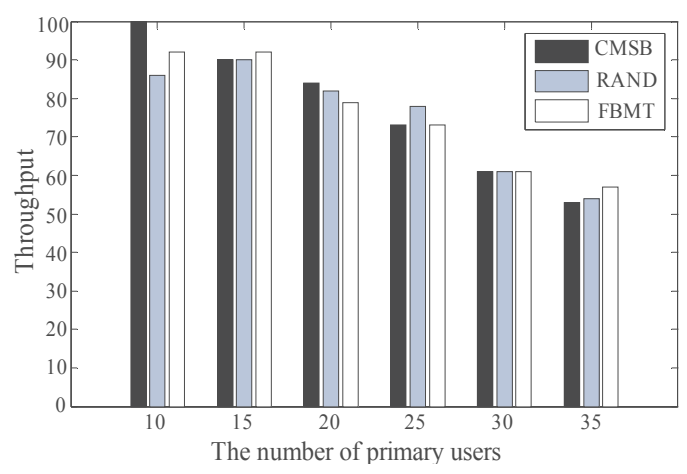

(a) $N=30, K=15, b_{i, k}=1$

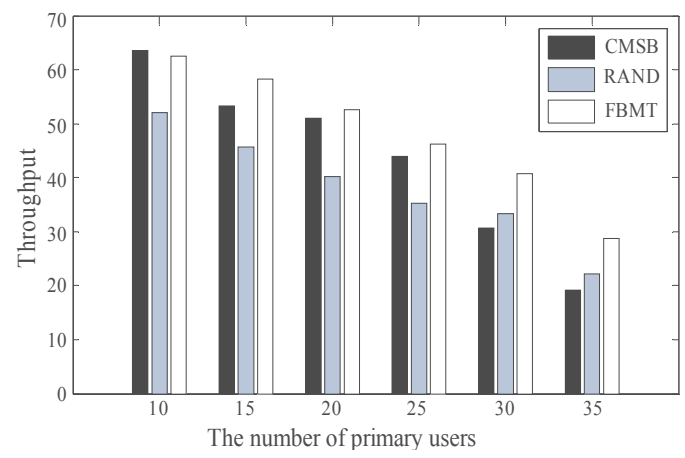

(b) $N=30, K=15, b_{i, k} \in[0,1]$

Figure 3. The number of primary users vs. system throughput. 


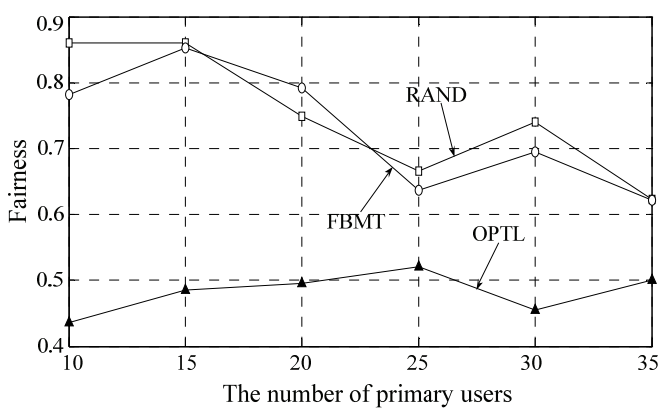

(a) $N=30, K=15, b_{i, k}=1$

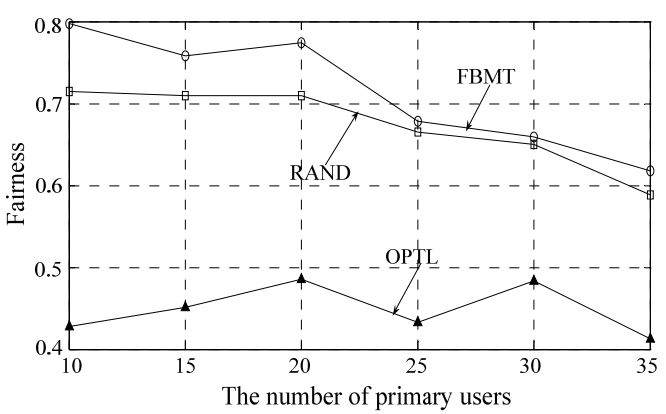

(b) $N=30, K=15, b_{i, k} \in[0,1]$

Figure 4. The number of primary users vs. fairness.

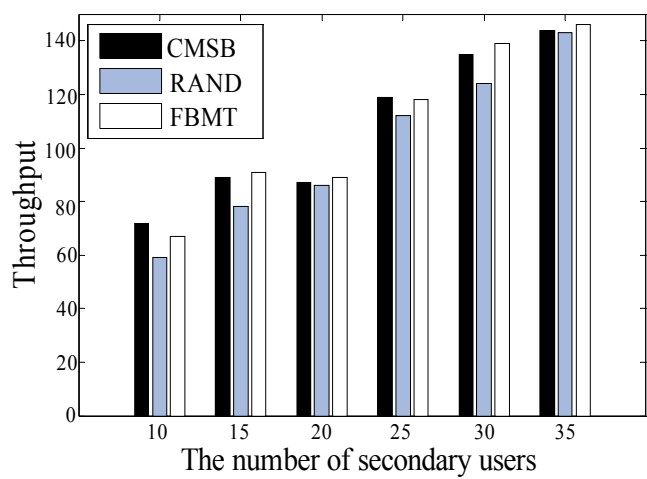

(a) $M=20, K=15, b_{i, k}=1$

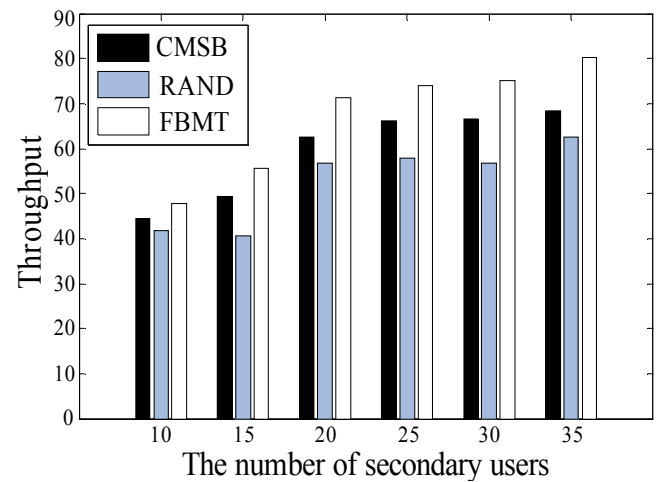

(b) $M=20, K=15, b_{i, k} \in[0,1]$

Figure 5. The number of secondary users vs. system throughput.

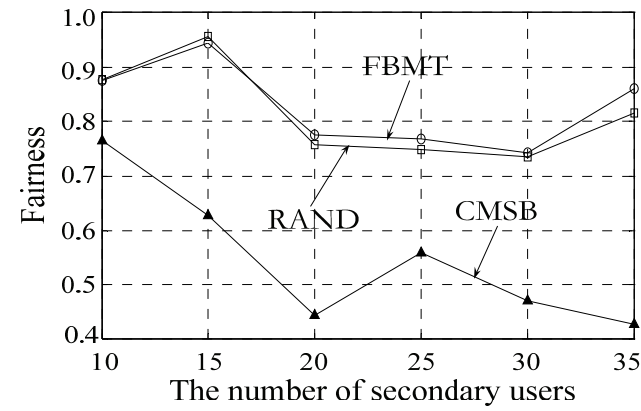

(a) $M=20, K=15, b_{i, k}=1$

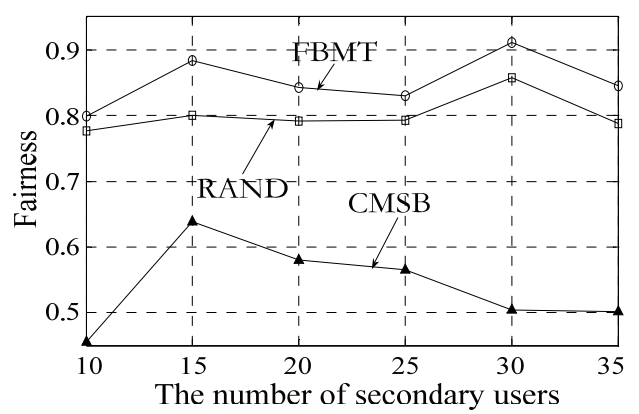

(b) $M=20, K=15, b_{i, k} \in[0,1]$

Figure 6. The number of secondary users vs. fairness.

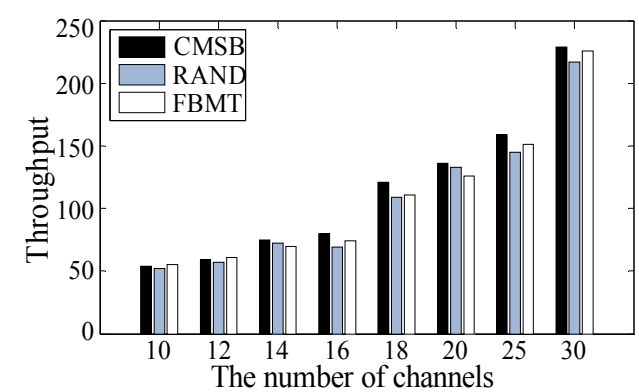

(a) $M=15, K=20, b_{i, k}=1$

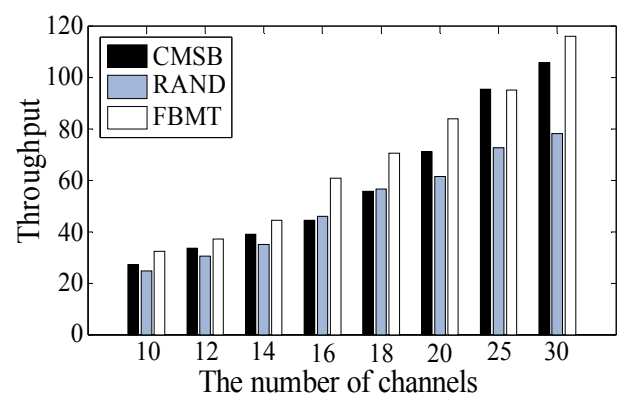

(b) $M=15, K=20, b_{i, k} \in[0,1]$

Figure 7. The number of spectrums vs. throughput. 


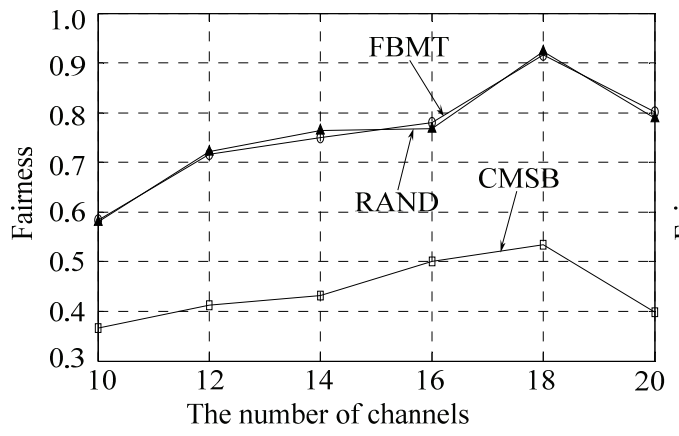

(a) $M=15, K=20, b_{i, k}=1$

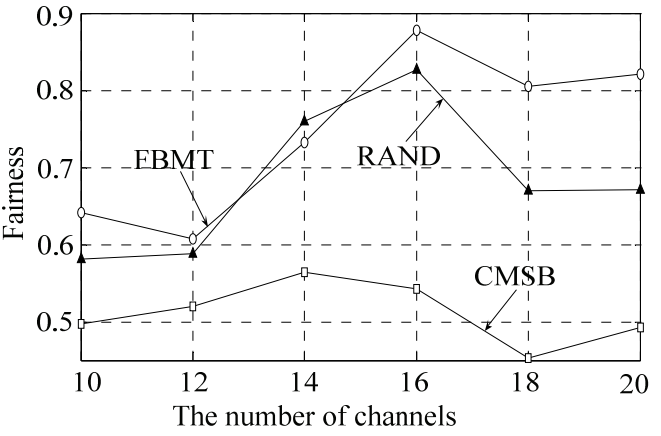

(b) $M=15, K=20, b_{i, k} \in[0,1]$

Figure 8. The number of spectrums vs. fairness.

\section{Conclusions}

In this paper, we explore the tradeoff in spectrum utilization and interference mitigation in open spectrum system. We develop a new graph-theoretical model to characterize the spectrum access problem under a number of different optimization functions, taking into account heterogeneity in both spectrum availability, reward and interference constraint.

We then propose an algorithm where each user can opportunistically utilize its available spectrum. Experimental results confirm that our algorithm significant benefits most in system throughput and also have a good performance in fairness.

\section{Acknowledgements}

This work was supported in part by National 863 Projects of China (2009AA01Z205), Fund of National Laboratory (P080010) and Program for New Century Excellent Talents in University (NCET070339).

\section{References}

[1] S. W. Ellingson, "Spectrum Occupancy at VHF: Implications for Frequency-Agile Cognitive Radios," Proceedings of IEEE Vehicle Technologies Conference, Vol. 9, No. 2, 2005, pp. 1379-1382.

[2] M. A. McHenry, "NSF Spectrum Occupancy Measurements Project Summary," Shared Spectrum Company Report, Vienna, 2005.

[3] M. McHenry, E. Livsics, T. Nguyen and N. Majumdar, "XG Dynamic Spectrum Access Field Test Results," IEEE Communications Magazine, Vol. 6, No. 45, 2007, pp. 51-57.
[4] J. Mitola and G. Q. Maguire, "Cognitive Radio: Making Software Radios More Personal," IEEE Personal Communications, Vol. 8, No. 6, 1999, pp. 13-18.

[5] H. Zheng and C. Peng, "Collaboration and Fairness in Opportunistic Spectrum Access," Proceedings of the 2005 IEEE International Conference on Communications (ICC'05), Seoul, Korea, 2005, pp. 3132-3136.

[6] C. Peng, H. Zheng and B. Y. Zhao, "Utilization and Fairness in Spectrum Assignment for Opportunistic Spectrum Access," Mobile Networks and Applications, Vol. 11, No. 4, 2006, pp. 555-576.

[7] J. Zhao, H. Zheng and G. Yang, "Distributed Coordination in Dynamic Spectrum Allocation Networks," Proceedings of the 2005 1st IEEE International Symposium on New Frontiers in Dynamic Spectrum Access Networks (DySPAN'05), Baltimore, 2005, pp. 259-268.

[8] L. Cao and H. Zheng, "Distributed Spectrum Allocation Via Local Bargaining," Proceedings of the 2nd Annual IEEE Communications Society Conference on Sensor and Ad Hoc Communications and Networks, Santa Clara, 2005, pp. 475-486.

[9] X. Liu and W. Wang, "On the Characteristics of Spectrum-Agile Communication Networks," Proceedings of the 2005 1st IEEE International Symposium on New Frontiers in Dynamic Spectrum Access Networks (DySP$\left.A N^{\prime} 05\right)$, Baltimore, 2005, pp. 214-223.

[10] W. Wang and X. Liu, "List-Coloring Based Spectrum Allocation for Open-Spectrum Wireless Networks," Proceedings of the IEEE International Symposium on Vehicular Technology (VTC'05-Fall), Dallas, 2005, pp. 690-694.

[11] Y. Xing, R. Chandramouli, S. Mangold and S. N. Shankar, "Analysis and Performance Evaluation of a Fair Spectrum Access Protocol for Open Spectrum Wireless Networks," Proceedings of the 2005 IEEE International Symposium on Communications (ICC'05), Seoul, Korea, 2005, pp. 1179-1183. 